\title{
On the Evaluation of Vacuum Energy Density in Quantum Field Theory
}

\author{
Ervin Goldfain \\ Research Scholar, Ronin Institute, Montclair, New Jersey 07043 \\ Email: ervin.goldfain@ronininstitute.org
}

\begin{abstract}
The textbook analysis of vacuum energy density (VED) in flat spacetime follows from Pauli's lectures of 1951, in which quantum vacuum is modeled as a reservoir of free harmonic oscillators. In his lectures, Pauli shows that deriving a nearly vanishing VED is contingent upon fulfilling three corollary conditions called polynomial-in-mass-constraints. The goal of this work is to evaluate Pauli's constraints against the Standard Model parameters and the Higgs mechanism of spontaneous symmetry breaking.
\end{abstract}

Key words: zero-point energy density, vacuum energy density, Standard Model parameters, mass spectrum, Renormalization Group flow, Higgs mechanism, cosmological constant problem.

\section{Pauli's VED model and its constraints}

According to [1-2], the vacuum energy density (VED) of a relativistic quantum field theory (QFT) on flat spacetime may be computed from

$$
\rho_{V}=\sum_{n}\left\{c_{n} \int \frac{d^{3} k}{(2 \pi)^{3}} \frac{1}{2} \hbar \omega_{n}(k)\right\}
$$

In this model, VED is considered a reservoir of free quantum harmonic oscillators and (1) is the integral of the zero-point energy carried over all momenta. While the bosonic terms 
in (1) are positive, the fermion terms are negative, hinting to a mutual cancellation of the overall VED. The coefficient in front of the integral is given by

$$
c_{n}=(-1)^{2 S_{n}} \overline{g_{n}}
$$

where $n$ denotes the index of particle species. The spin-dependent degeneracy factor $\bar{g}_{n}$ for massive particles of spin $S_{n}$ assumes the form

$$
\overline{g_{n}}=2 S_{n}+1
$$

All massless particles are defined instead by $\bar{g}_{n}=2$. The degeneracy factor includes an additional factor of 2 accounting for distinct particle-antiparticle states and an additional factor of 3 accounting for color. It can be shown that, using the relativistic dispersion relation

$$
\omega_{n}(k)=\sqrt{m_{n}^{2}+k^{2}}
$$

the total VED vanishes identically (or stays finite) upon imposing three so-called polynomial-in-mass constraints presented as [1-2]

$$
\begin{gathered}
\sum_{n} c_{n}=0 \\
\sum_{n} c_{n} m_{n}^{2}=0 \\
\sum_{n} c_{n} m_{n}^{4}=0
\end{gathered}
$$


along with a fourth logarithmic-in-mass condition

$$
\rho_{V}=\frac{\hbar}{64 \pi^{2}} \sum_{n} c_{n} m_{n}^{4} \ln \left(m_{n}^{2} / \mu^{2}\right)
$$

in which $\mu$ stands for the Renormalization Group (RG) scale.

We close this section by noting that the VED model (1)-(5) may be generalized to a framework where the set $(5)$ is tied to the finiteness and Lorentz invariance of the VED stress-energy tensor [2].

\section{Pauli's analysis versus Standard Model requirements}

As Pauli already remarks in [1], simultaneously enforcing (5a)-(5c) boils down to a highlyimprobable cancellation of terms including weighted powers of particle masses. Years after Pauli's lectures of 1951, one can trivially check that (5a)-(5c) fail to hold when applied to the Standard Model (SM) masses evaluated near the electroweak (EW) scale. The goal of this section is to look deeper into this mismatch and its implications for the cosmological constant problem.

Near the EW scale, symmetry breaking connects particle masses with the vacuum expectation value of the Higgs boson $(\mathrm{v} \approx 246 \mathrm{GeV})$ via [3]

$$
\begin{gathered}
m_{H}=\sqrt{2 \lambda} \mathrm{v} \\
m_{W}=\frac{g_{2}}{2} \mathrm{v} \\
m_{Z}=\frac{\sqrt{g_{1}^{2}+g_{2}^{2}}}{2} \mathrm{v}
\end{gathered}
$$




$$
m_{f}=\frac{y_{f}}{\sqrt{2}} \mathrm{v}
$$

where $g_{1}, g_{2}$ is the pair of gauge charges of the EW model, $\lambda$ is the quartic interaction coupling of the Higgs boson and $y_{f}$ the Yukawa coupling of fermions.

Direct substitution of (6) in (5) turns the Pauli's constraints into conditions written in terms of interaction couplings. This brings us to the observation that, in general, interaction couplings are not independent parameters. Specifically,

a) Near the EW scale set by $v$, the so-called "sum-of-squares" requirement amounts to the relationship [4]

$$
2 \lambda+\frac{g_{2}^{2}}{4}+\frac{g_{1}^{2}+g_{2}^{2}}{4}+\sum_{f} \frac{y_{f}^{2}}{2}=1
$$

b) Away from the EW scale, all couplings flow with $\mu$. The flow can be either perturbative or non-perturbative and is prone to amplify deviations from Pauli's constraints (5).

Regarding point b), it is fair to state that the one of the least understood aspects of RG equations lies within the Higgs sector and is rooted in the so-called gauge-hierarchy problem of the SM [Appendix A]. Close to this problem is the issue of whether the Standard Model remains valid all the way up to the Planck scale $\left(M_{P l}\right)$. This question is non-trivial because it hinges on how $\lambda$ behaves at high energies. Competing trends are at work here, namely [7]:

1) Radiative corrections from top quarks $d r o p \lambda$ at higher scales, while those from the self-interacting Higgs grow $\lambda$ at higher scales. 
2) If $\lambda\left(M_{E W}\right)$ is too large, the Higgs loops dominate and $\lambda$ diverges at some intermediate scale called the Landau pole. However, if $\lambda\left(M_{E W}\right)$ is too small, the top loops dominate, $\lambda$ runs negative at some intermediate scale which, in turn, makes the potential unbounded from below and destabilizes the vacuum.

To further reinforce the fact that the SM couplings are mutually dependent observables, consider next the RG flow of Pauli's constraints. It is described by the equations [2]

$$
\begin{aligned}
& \frac{d}{d t}\left(\sum_{n} c_{n} m_{n}^{2}\right)=2\left(\sum_{n} c_{n} m_{n}^{2} \gamma_{n}\right) \\
& \frac{d}{d t}\left(\sum_{n} c_{n} m_{n}^{4}\right)=4\left(\sum_{n} c_{n} m_{n}^{4} \gamma_{n}\right)
\end{aligned}
$$

in which

$$
t=\ln \left(\mu / \mu_{0}\right)
$$

and where the mass renormalization factor is given by

$$
\gamma_{n}=\frac{1}{m_{n}} \frac{\partial m_{n}}{\partial t}
$$

By (6), near the EW scale, one arrives at

$$
m_{n}=g_{n} \mathrm{v} \Rightarrow \gamma_{n}=\frac{1}{g_{n}} \frac{\partial g_{n}}{\partial t}=\frac{\beta_{n}\left(g_{n}\right)}{g_{n}}
$$


with the generic SM coupling symbolically written as $g_{n}=\left\{\lambda, g_{1}, g_{2}, g_{3}, y_{f, n}\right\}$, in which $g_{3}$ denotes the strong interaction charge $\left(g_{3}=g_{Q C D}\right)$. The RG equations defining the flow of $g_{n}$ can be perturbatively represented as [6]

$$
\beta(g)=\frac{1}{(4 \pi)^{2}} \beta^{(1)}(g)+\frac{1}{(4 \pi)^{4}} \beta^{(2)}(g)+\ldots
$$

Each term of the series (12) may be factorized in the form

$$
\begin{gathered}
\beta^{(i)}\left(g_{n}\right)=g_{n} \overline{\beta^{(i)}}\left(g_{n}\right) \\
\beta_{f}^{(i)}=y_{f} \overline{\beta_{f}^{(i)}} \\
\beta_{\mathrm{v}}^{(i)}=\mathrm{v} \overline{\beta_{\mathrm{v}}^{(i)}}
\end{gathered}
$$

According to [6], the one-loop expressions for the beta functions of (13)-(15) are given by

$$
\begin{gathered}
\bar{\beta}^{(1)}\left(g_{n}\right)=-b_{n} g_{n}^{2}, n=1,2,3 \\
\bar{\beta}_{f}^{(1)}=f_{f}\left(y_{f}, g_{1}, g_{2}, g_{3}\right) \\
\bar{\beta}_{\mathrm{v}}^{(1)}=f_{\mathrm{v}}\left(y_{f}, g_{1}, g_{2}\right)
\end{gathered}
$$

Inserting (12)-(18) into (8) shows that the flow of Pauli's constraints represents a multidimensional system of coupled nonlinear differential equations, whose long-run evolution is prone to end on an aperiodic attractor. This is consistent with the observation that multidimensional RG flows do not typically settle on fixed points, but 
rather on limit cycles, quasiperiodic tori or strange attractors [8-10]. The key observation here is that the fixed points of truly conformal invariant theories describe exclusively massless particles. By contrast, the SM is a nonlinearly interacting theory of massive particles, substantially deviating from the simplistic picture of harmonic oscillators radiating freely in empty space.

\section{Concluding remarks}

Our analysis indicates that, insofar the SM is concerned, Pauli's model breaks Lorentz invariance of the VED stress-energy tensor put forward in [2]. Together with the considerations of Appendix B, these findings suggest that basing the cosmological constant problem on Pauli's model may need to be revised.

\section{Appendix A}

\section{The gauge hierarchy problem}

The EW symmetry of the SM is broken by a scalar field having the following doublet structure [7] :

$$
\Phi=\left(\begin{array}{c}
G^{+} \\
1 / \sqrt{2}\left[(H+\mathrm{v})+i G^{0}\right]
\end{array}\right)
$$

Here, $G^{+}$and $G^{0}$ represent the charged and neutral Goldstone bosons arisen from spontaneous symmetry breaking, $H$ is the SM Higgs boson, $\mathrm{v} \approx O\left(M_{E W}\right)=246 \mathrm{GeV}$ is the Higgs vacuum expectation value and $M_{E W}$ the Fermi scale. Symmetry breaking is caused 
by the Higgs potential, whose form satisfies the requirements of renormalizability and gauge-invariance:

$$
V=\mu_{H}^{2} \Phi^{+} \Phi+\lambda\left(\Phi^{+} \Phi\right)^{2}
$$

with $\lambda \approx O(1)$ and $\mu_{H}^{2} \approx O\left(M_{E W}^{2}\right)$. A vanishing quartic coupling $(\lambda=0)$ represents the critical value that separates the ordinary EW phase from an unphysical phase where the Higgs field assumes unbounded values. Likewise, the coefficient $\mu_{H}^{2}$ plays the role of an order parameter whose sign describes the transition between a symmetric phase and a broken phase. Minimizing the Higgs potential yields a vacuum expectation value given by:

$$
\mathrm{v}^{2}=-\left(\mu_{H}^{2} / \lambda\right)
$$

where the physical mass of the Higgs is:

$$
m_{H}^{2}=\left|-2 \lambda v^{2}\right|=2 \mu_{H}^{2}
$$

The renormalized mass squared of the Higgs scalar contains two contributions:

$$
\mu_{H}^{2}=\mu_{b}^{2}+\Delta \mu_{H}^{2}
$$

in which $\mu_{b}^{2}$ represents the ultraviolet (bare) value. This mass parameter picks up quantum corrections $\Delta \mu_{H}^{2}$ that depend quadratically on the ultraviolet cutoff $\Lambda$ of the theory. Consider, for example the contribution of radiative corrections to $\mu_{H}^{2}$ from top quarks. The complete one-loop calculation of this contribution reads: 


$$
\Delta \mu_{H}^{2}=\frac{N_{c} \lambda_{t}^{2}}{16 \pi^{2}}\left[-2 \Lambda^{2}+6 M_{t}^{2} \ln \left(\frac{\Lambda}{M_{t}}\right)+\ldots\right]
$$

in which $\lambda_{t}$ and $M_{t}$ are the Yukawa coupling and mass of the top quark. If the bare Higgs mass is set near the cutoff $\mu_{b}^{2}=O\left(\Lambda^{2}\right)=O\left(M_{P l}^{2}\right)$, then $\Delta \mu_{H}^{2} \approx-10^{35} \mathrm{GeV}$. This large correction must precisely cancel against $\mu_{b}^{2}$ to protect the EW scale. This is the root cause of the fine-tuning (or the gauge hierarchy) problem, which boils down to the implausible requirement that $\mu_{b}^{2}$ and $\Delta \mu_{H}^{2}$ should offset each other to an unnaturally large number of decimal places.

\section{$\underline{\text { Appendix B }}$}

\section{Pauli's model and the cosmological constant problem}

A cursory glance at (4) reveals that, for large momenta $k>>m_{n}$, the oscillator frequency may be approximated as $\omega_{n}(k) \approx k$, in which case the integral (1) diverges. To isolate the effect of high-energy contributions in the computation of VED, Pauli inserts an ultraviolet (UV) cutoff $\Lambda$ in the integral of (1), whose expansion yields

$$
\int_{0}^{\Lambda} d^{3} k \sqrt{m_{n}^{2}+\Lambda^{2}}=\pi\left\{\Lambda^{4}+m_{n}^{2} \Lambda^{2}+\frac{m_{n}^{4}}{8}-\frac{1}{2} m_{n}^{4} \ln \left(2 \Lambda / m_{n}\right)\right\}+O\left(\Lambda^{-2}\right)
$$

It is apparent that (B1) is quartically divergent as the UV cutoff approaches the Planck region of scales $\left(\Lambda=O\left(M_{P l}\right)>>m_{n}\right)$. To regularize (B1) one follows the general renormalization prescription of QFT, according to which one starts with a bare Lagrangian and a cutoff dependent bare VED in the form 


$$
\rho_{b}=\rho_{b}(\Lambda)
$$

As a result, the renormalized or effective VED is given by [5]

$$
\rho_{e f f, \mathrm{v}}=\rho_{b}(\Lambda)+c \Lambda^{4}
$$

where $c$ stands for some numerical constant. Astrophysical observations from type I supernovae and from the cosmic microwave background (CMB) radiation show that

$$
\rho_{e f f, v}^{1 / 4} \approx 2 \times 10^{-3} \mathrm{eV}
$$

Since experiments have confirmed that SM is valid at least up to an energy scale of $O\left(1 \mathrm{TeV}=10^{12} \mathrm{eV}\right)$, one may reasonably assume that the UV cutoff can be placed around this scale $\left(\Lambda=O\left(10^{12} \mathrm{eV}\right)\right)$. Combined use of (B3) and (B4) leads to

$$
\left(2 \times 10^{-3} \mathrm{eV}\right)^{4}=\rho_{b}(\Lambda)+c\left(10^{12} \mathrm{eV}\right)^{4}
$$

Thus, the bare value of the cosmological constant evaluated at the cutoff must be chosen so that it cancels out a contribution on the order of $10^{48} \mathrm{eV}^{4}$ and leaves a contribution on the order of $10^{-12} \mathrm{eV}^{4}$. This requires an unnatural fine-tuning of the cosmological constant on the order of 60 decimal places, which lies at the heart of the cosmological constant problem.

\section{$\underline{\text { References }}$}

1. Pauli W., "Pauli Lectures on Physics: Vol 6, Selected Topics in Field Quantization", MIT Press, 1971. 
2. https://arxiv.org/pdf/1610.07264.pdf

3. Cheng T.P. and Li L. F., "Gauge theory of elementary particle physics", Clarendon Press, Oxford, 1989.

4. $\underline{\text { https://arxiv.org/pdf/1305.4208.pdf }}$

5. Maggiore M., "A Modern Introduction to Quantum Field Theory”, Oxford Univ. Press, 2006.

6. https://arxiv.org/pdf/1703.01523.pdf

7. https://arxiv.org/pdf/1205.6497.pdf

8. https://arxiv.org/pdf/hep-th/0304178.pdf

9. Available at the following site:

https://www.researchgate.net/publication/228625050 Chaotic Dynamics of the Re normalization Group Flow and Standard Model Parameters

10. https://www.worldscientific.com/doi/abs/10.1142/So218127408020756 